\title{
False-positive IgM for CMV in pregnant women with autoimmune disease: a prognostic index of poor pregnancy outcome
}

\begin{abstract}
Our aims were to assess the frequency of false-positive $\operatorname{IgM}$ antibodies for cytomegalovirus in pregnant women with autoimmune diseases and in healthy women (controls) and to determine their relationship with pregnancy outcome. Data from 265 pregnancies in 236 patients with autoimmune diseases and from 265 pregnancies in 243 controls were assessed. When positive IgM for cytomegalovirus was detected, IgG avidity, cytomegalovirus isolation and polymerase chain reaction for CMV-DNA in maternal urine, blood and amniotic fluid samples were performed in order to identify primary infection or false positivity. A statistically significantly higher rate of false-positive IgM was found in pregnancies with autoimmune diseases $(10 \%)$ in comparison with controls $(0.7 \%)$. A worse pregnancy outcome was observed among patients with autoimmune disease and false cytomegalovirus $\operatorname{IgM}$ in comparison with those without false positivity: earlier week of delivery $(p<0.0001)$, lower neonatal birth weight $(p<0.0001)$ and neonatal birth weight percentile $(\mathrm{p}<0.0001)$, higher rate of intrauterine growth restriction $(\mathrm{p}=0.004)$ and babies weighing less than $2000 \mathrm{~g}(\mathrm{p}<0.0001)$ were encountered. The presence of false cytomegalovirus $\operatorname{IgM}$ in patients with autoimmune diseases could be used as a novel prognostic index of poor pregnancy outcome: it may reflect a non-specific activation of the immune system that could negatively affect pregnancy outcome.
\end{abstract}

Volume 2 Issue 2 - 2015

Tabacco S,' Lanzone A,' Botta A,' Salvi

S,' Martino C,' Del Sordo G,' Garufi C,' Lanzone A,' Sara De Carolis'

'Department of Obstetrics and Gynecology, Catholic University of the Sacred Heart, Rome, Italy

${ }^{2}$ Department of Internal Medicine and Medical Specialities, Rheumatology Unit, Sapienza University of Rome, Rome, Italy

Correspondence: Sara De Carolis, Coordinatrice Polo Donna - Clinical Governance, Dipartimento della Salute della Donna, della Vita Nascente e del Bambino, Catholic University "Sacro Cuore" - Policlinico Gemelli, Largo A Gemelli 800168 Rome, Italy, Tel 39(06)30I56774, 393384929904, Fax 39(06)305 I I60, Emailsara.decarolis@rm.unicatt.it

Received: January 12, 2015 | Published: June 05, 2015

\section{Introduction}

The relationship between autoimmune diseases and infections is an interesting and complex issue. Autoimmunity is a multifactorial process in which genetics, ormonals, immunologics and infectious factors act like pieces of a mosaic in the genesis of autoimmune diseases. Infections may be considered as a trigger factor of the onset or exacerbation of autoimmune diseases, inducing a non-specific activation of lynphocytes B. ${ }^{1}$ In predisposed subjects, antigenic similarity between infectious agents and host tissues might result, by a mechanism of molecular mimicry, in an immune response to shared determinants, resulting in disease. ${ }^{1-6}$ Moreover an immunosuppressive status due to the use of steroids or cytotoxic drugs in these patients may contribute to the onset of opportunistic infections or reactivation of infectious agents. ${ }^{7}$ Finally, in patients with autoimmune disease, the serological diagnosis of viral infection may be distorted by production of specific antibodies without acute infection that can leads in false-positive results of the common tests used to diagnose infections. Clinicians are aware that systemic lupus erythematosus (SLE), anti phospholipid syndrome (APS) and rheumatoid arthritis are often associated with false-positive serological tests for CMV and other infections, such as HIV, rubella, toxoplasmosis, Lyme disease, and syphilis. ${ }^{8}$ The false-positivity of anti-CMV IgM antibodies (false CMV IgM) in healthy pregnancies is not correlated to poor pregnancy outcome. Furthermore, in pregnancies complicated by autoimmune disease, this condition is associated with particular clinical implications.

The aim of this study is to analyze the significance of false CMV IgM in pregnant women with autoimmune disease. Firstly, we investigated the frequency of false CMV IgM in pregnant women with autoimmune disease in comparison with healthy pregnant women. Secondly, we evaluated the relationship between false CMV IgM and poor pregnancy outcome.

\section{Patients and methods}

In order to assess the frequency of false-positive results of the screening test for CMV in pregnant women with autoimmune disease, 262 ongoing singleton pregnancies in 236 patients with autoimmune diseases were retrospectively assessed as the study group Table 1. Patients were followed in our tertiary referral centre from 2000 to 2012 . Sixty-six pregnancies $(25.1 \%)$ were complicated by primary APS, according to the revised classification criteria (Sydney 2004); 16 pregnancies $(6.1 \%)$ by secondary APS (associated with other autoimmune diseases), of which nine pregnancies were associated with SLE, according to the ACR classification criteria; 85 pregnancies (32.4\%) were complicated by SLE, and 95 pregnancies $(36.2 \%)$ by other autoimmune diseases (rheumatoid arthritis, Sjogren disease, undifferentiated connective tissue disease, autoimmune thyroiditis, idiopathic thrombocytopenic purpura, myasthenia gravis, celiac disease, autoimmune vasculitis and multiple sclerosis). According to their disease, patients received specific treatment (low-dose aspirin, heparin, immunosuppressive drugs, ant malarial drugs, etc.); they were serially followed by ultrasound examinations and laboratory tests and admitted to our high-risk pregnancy unit when necessary. In addition, 265 ongoing singleton pregnancies in 243 healthy women were assessed as the control group.

Each woman in both groups was systematically screened for anti-CMV IgG and IgM during preconception evaluation and/or at the beginning of pregnancy. Serum samples were tested for CMV antibodies using commercial microparticle enzyme immunoassays. The test for the detection of anti-CMV IgM contains the RF absorbent; this eliminates false-positive results caused by certain rheumatoid factors as well as false-negative results that can be caused by a high concentration of virus-specific IgG. The test specificity is $100 \%$ and sensitivity is $95 \%$. To better distinguish primary CMV infection from false-positive results, pregnant women with positive anti-CMV IgM 
were also tested with an anti-CMV IgG avidity assay; CMV isolation in maternal urine, vaginal and oropharyngeal samples; CMV isolation in amniotic fluid samples, obtained by amniocentesis, if gestational age was less than 20 weeks; and polymerase chain reaction (PCR) for CMV-DNA detection in maternal urine and amniotic fluid samples.

Table I Characteristics of the study group

\begin{tabular}{|ll|}
\hline Number of pregnancies $\mathbf{N}=\mathbf{2 6 2}(\%)$ & Autoimmune disease \\
\hline $66(25.1)$ & Primary APS \\
$16(6.1)$ & Secondary APS \\
$85(32.4)$ & SLE \\
$95(36.2)$ & Others \\
\hline
\end{tabular}

${ }^{a}$ Others: rheumatoid arthritis, sjogren disease, undifferentiated connective tissue disease, autoimmune thyroiditis, idiopathic thrombocytopenic purpura, myasthenia gravis, celiac disease, auto immune vasculitis, multiple sclerosis

The avidity index (AI) was expressed as follows: percentage of AI $1 / 4$ (absorbance result of CMV per well with/without urea wash) 100. High $\operatorname{IgG}$ avidity (AI>30\%) is considered as non-recent primary infection (more than 3 months) and low IgG avidity $(\mathrm{AI}<20 \%)$ is suggestive of recent primary infection (less than 3 months). Intermediate IgG avidity (AI $20-30 \%$ ) is more difficult to interpret.

Anti-CMV IgM positivity associated with high IgG avidity, negative viral isolation on maternal urine samples, vaginal smears, oropharyngeal and amniotic fluid samples, and negative PCR for CMV-DNA on amniotic fluid and maternal urine samples in more recent cases was identified as a false-positive result for anti-CMV IgM. In order to investigate the possible relationship between false CMV IgM and pregnancy outcome in patients with autoimmune diseases, ongoing pregnancies were included in the analysis. The week of delivery, the birth weight, the birth weight percentile, the rate of preterm delivery (37 weeks of gestation), intrauterine growth restriction (IUGR) (defined as a birth weight percentile $<10$ ) and babies weighing less than $2500 \mathrm{~g}$ or less than $2000 \mathrm{~g}$ were all considered indicators of pregnancy outcome.

\section{Statistical analysis}

Normally distributed continuous variables were compared using a two-sample Student t test. Cross-tabulation and Chi-square (with Yates' continuity correction) were used to examine the relationship between nominal variables. $\mathrm{p}<0.05$ was considered statistically significant. The odds ratio (OR) and the $95 \%$ confidence interval (CI) were calculated to measure the clinical effect of false-positive CMV IgM results on pregnancy outcome, in terms of IUGR and babies with birth weight less than $2000 \mathrm{~g}$. SPSS software version 12 was used to calculate accuracy indices such as negative predictive value (NPV) and positive predictive value (PPV), in order to evaluate the proportion of women who did not report preterm delivery, IUGR, neonatal birth weight $2500 \mathrm{~g}$ and neonatal birth weight $2000 \mathrm{~g}$ and who were negative for the test, and the proportion of women who reported preterm delivery, IUGR, neonatal birth weight $2500 \mathrm{~g}$ and neonatal birth weight $2000 \mathrm{~g}$ and who were positive for the test, respectively.

\section{Results}

Out of 262 pregnancies of the study group, 27 were judged as having false CMV IgM (10\%), and none of these patients had any manifestation of CMV infection. ${ }^{8-10}$ Out of 262 pregnancies in the control group, two pregnancies $(0.7 \%)$ showed false CMV IgM results. The rate of false CMV IgM was statistically significantly higher in pregnancies with autoimmune disease in comparison with that of the control group $(\mathrm{p}<0.0001)$. The rate of positive anti-CMV IgG was similar in the two groups, being $79 \%$ in the study group and $75 \%$ in the control group. The prevalence of false-positive results for different autoimmune diseases was: $15 \%$ in primary APS, $11 \%$ in secondary APS, $41 \%$ in SLE, and 33\% in pregnancies complicated by other autoimmune diseases. Pregnancy outcome results are shown in Tables $2 \& 3$. In the study group, the mean week of delivery was 37.7 , the mean neonatal birth weight was $2888 \mathrm{~g}$, the mean neonatal birth weight percentile was 45 , the rate of IUGR was $12 \%$, the rate of babies weighing at birth $<2500 \mathrm{~g}$ was $23 \%$ and the rate of babies weighing $<2000 \mathrm{~g}$ was $8 \%$. In the control group, the mean week of delivery was 39.6 , the mean neonatal birth weight was $3287 \mathrm{~g}$, the mean neonatal birth weight percentile was 54 , the rate of IUGR was $5 \%$, the rate of babies weighing at birth $<2500 \mathrm{~g}$ was $3 \%$, and the rate of babies weighing $<2000$ g was $1 \%$.

Table 2 Pregnancy outcome in women with autoimmune disease (study group) and in healthy pregnant women (control group)

\begin{tabular}{|c|c|c|c|}
\hline & $\begin{array}{l}\text { Study group } \\
(n=262)\end{array}$ & $\begin{array}{l}\text { Control group } \\
(n=265)\end{array}$ & $\mathbf{p}$ \\
\hline \multicolumn{4}{|c|}{ At term deliveries ${ }^{\mathrm{a}}$} \\
\hline $\mathrm{n}$ & 155 & 240 & $<0.0001$ \\
\hline$\%$ & 59 & 91 & \\
\hline \multicolumn{4}{|c|}{ Preterm deliveries ${ }^{\mathrm{b}}$} \\
\hline $\mathrm{n}$ & 107 & 25 & $<0.0001$ \\
\hline$\%$ & 41 & 9 & \\
\hline \multicolumn{4}{|c|}{ Week of delivery } \\
\hline mean $\pm S D$ & $37.97 \pm 2.33$ & $39.6 \pm 1.73$ & $<0.0001$ \\
\hline \multicolumn{4}{|c|}{ Birth weight (g) } \\
\hline mean $\pm S D$ & $2888 \pm 612.63$ & $3286 \pm 481.69$ & $<0.0001$ \\
\hline \multicolumn{4}{|c|}{ Birth weight percentile } \\
\hline $\begin{array}{l}\text { mean } \pm S D \\
I G^{c}\end{array}$ & $44 \pm 24.99$ & $54.4 \pm 24.70$ & $<0.0001$ \\
\hline $\mathrm{n}$ & 31 & 13 & 0.004 \\
\hline$\%$ & 12 & 5 & \\
\hline \multicolumn{4}{|c|}{ Babies with birth weight $<2500 \mathrm{~g}$} \\
\hline $\mathrm{n}$ & 61 & 8 & \\
\hline$\%$ & 23 & 3 & $<0.0001$ \\
\hline \multicolumn{4}{|c|}{ Babies with birth weight $<2000 \mathrm{~g}$} \\
\hline $\mathrm{n}$ & 21 & 3 & $<0.0001$ \\
\hline$\%$ & 8 & 1 & \\
\hline
\end{tabular}

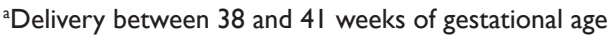

bDelivery $\leq 37^{\circ}$ weeks of gestational age

'Birth weight $<10$ th percentile for gestational age

In the study group a worse pregnancy outcome was observed among women with false CMV IgM in comparison with those without false-positive antibodies. In fact, earlier mean week of delivery (36.8 weeks versus 37.8 weeks, respectively, $\mathrm{p}=0.04)$, lower mean neonatal birth weight ( $2571 \mathrm{~g}$ versus $2925 \mathrm{~g}$ respectively, $\mathrm{p}=0.004)$ and lower mean neonatal birth weight percentile (34th versus 46th, respectively, $\mathrm{p}=0.002$ ) were encountered. Furthermore, a significantly higher rate of IUGR ( $26 \%$ versus $10 \%$, respectively, $\mathrm{p}=0.02$, OR 3.08 , CI $95 \%$, $1.18-8.03)$ and babies weighing less than $2000 \mathrm{~g}$ (19\% versus $7 \%$, $\mathrm{p}=0.05$, OR 3.11, CI 95\%, 1.04-9.30) was observed in pregnancies with false-positive antibodies compared with those without false-positive antibodies. A good pregnancy outcome was observed in two cases of false CMV-IgM in the control group. Both patients delivered at 41 weeks of gestation, neonatal weight was $3120 \mathrm{~g}$ (29th percentile) and $3650 \mathrm{~g}$ (73rd percentile), respectively. In order to evaluate the role of false positivity for CMV in SLE disease, we analyzed pregnancy outcome in the group of patients with SLE, excluding all cases with positive anti phospholipid antibodies $(n=85)$. Results are shown 
in Table 4. A poor pregnancy outcome was found in pregnancies with false positivity for CMV. Earlier mean week of delivery (35.6 weeks versus 38.4 weeks, respectively, $\mathrm{p}=0.0031$ ), lower mean neonatal birth weight ( $2187 \mathrm{~g}$ versus $2953 \mathrm{~g}$, respectively, $\mathrm{p}=0.0002)$ and lower mean neonatal birth weight percentile (24th versus 45 th, respectively, $p=0.0108)$ were encountered. The incidence of preterm delivery was higher in SLE pregnancies with false positivity for CMV than SLE without false positivity, but the difference did not reach the statistical significance. Focusing on the rate of IUGR, babies weighing $<2500 \mathrm{~g}$ and babies weighing $<2000 \mathrm{~g}$, the risk increased 11,7 and 5-fold, respectively, in comparison with the rate shown in the control group. NPV and PPV of false CMV IgM were calculated, as shown in Table 4. For IUGR, the NPV was $90 \%$ (PPV: $26 \%$ ), while for neonatal birth weight $2000 \mathrm{~g}$ NPV was $93 \%$ (PPV: $18 \%$ ).

Table 3 Pregnancy outcome in women with autoimmune disease (study group) with and without false positive anti-CMV IgM

\begin{tabular}{|c|c|c|c|}
\hline & Study group positive anti-CMV IgM (n=27) & $\begin{array}{l}\text { Study group negative } \\
\text { anti-CMV IgM }(n=235)\end{array}$ & $\mathbf{p}$ \\
\hline \multicolumn{4}{|c|}{ At-term deliveries ${ }^{a}$} \\
\hline $\mathrm{n}$ & 14 & $14 \mid$ & \\
\hline$\%$ & 52 & 60 & NS \\
\hline \multicolumn{4}{|c|}{ Preterm deliveries ${ }^{\mathrm{b}}$} \\
\hline $\mathrm{n}$ & 13 & 94 & \\
\hline$\%$ & 48 & 40 & NS \\
\hline \multicolumn{4}{|c|}{ Week of delivery } \\
\hline mean $\pm S D$ & $36.8 \pm 3.38$ & $37.8 \pm 2.17$ & 0.04 \\
\hline \multicolumn{4}{|c|}{ Birth weight(g) } \\
\hline mean $\pm S D$ & $2570.7 \pm 755.55$ & $2925.0 \pm 584.98$ & 0.004 \\
\hline \multicolumn{4}{|c|}{ Birth weight percentile } \\
\hline $\begin{array}{l}\text { mean } \pm S D \\
I^{\prime} G^{c}\end{array}$ & $34.1 \pm 25.70$ & $45.9 \pm 24.67$ & 0.02 \\
\hline $\mathrm{n}$ & 7 & 24 & \\
\hline$\%$ & 26 & 10 & 0.02 \\
\hline \multicolumn{4}{|c|}{ Babies with birth weight $<2500 \mathrm{~g}$} \\
\hline $\mathrm{n}$ & II & 50 & \\
\hline$\%$ & 30 & 21 & 0.03 \\
\hline \multicolumn{4}{|c|}{ Babies with birth weight $<2000 \mathrm{~g}$} \\
\hline $\mathrm{n}$ & 5 & 16 & \\
\hline$\%$ & 19 & 7 & 0.05 \\
\hline
\end{tabular}

a Delivery between 38 and 41 weeks of gestational age

${ }^{\mathrm{b}}$ Delivery $\leq 37^{\circ}$ weeks of gestational age

'Birth weight $<10$ th percentile for gestational age

Table 4 Pregnancy outcome in women with systemic lupus erythematosus without positivity of antiphospholipid antibodies

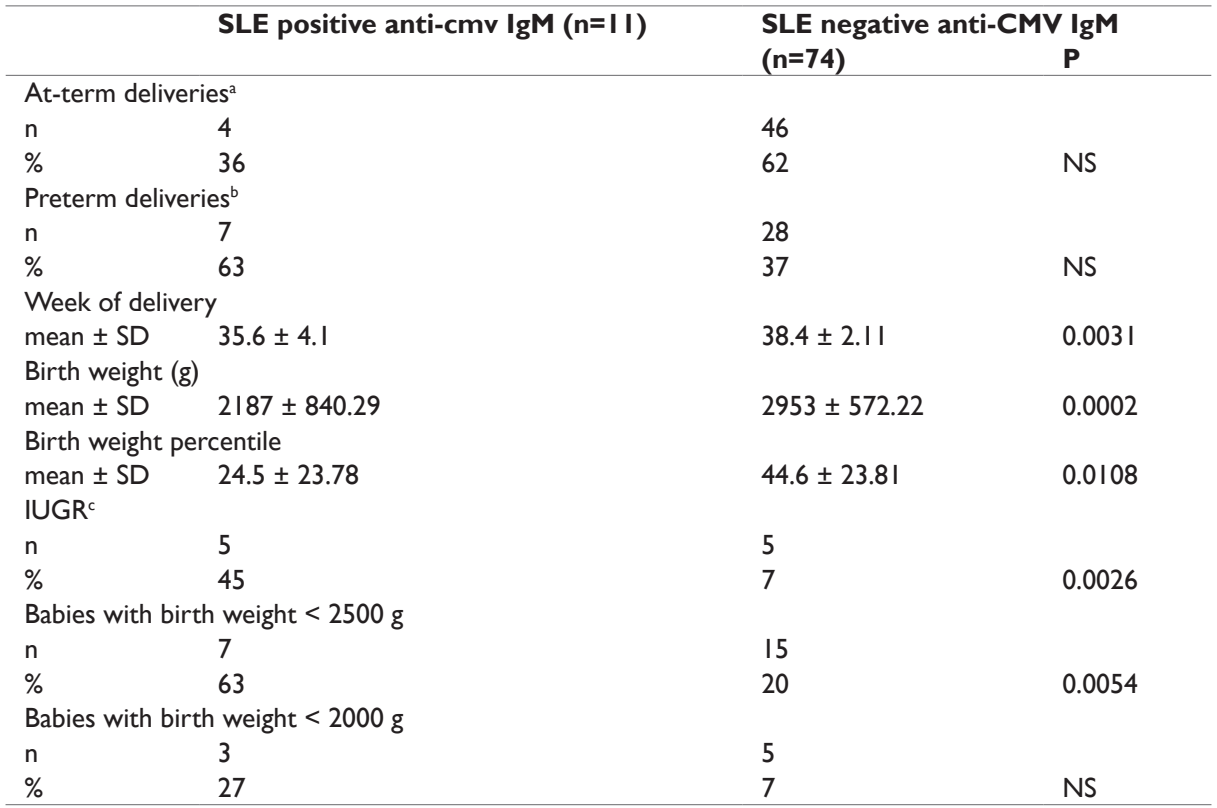

aDelivery between 38 and 41 weeks of gestational age

${ }^{b}$ Delivery $\leq 37^{\circ}$ weeks of gestational age

'Birth weight $<10$ th percentile for gestational age 


\section{Discussion}

The issue of false-positive tests for infectious agents was introduced and discussed in a number of studies. ${ }^{9-13}$ The Venereal Disease Research Laboratory (VDRL) test was falsely positive in autoimmune disease, such as SLE and APS, without clinical or serologic evidence of syphilis. ${ }^{14}$ False VDRL can be caused by the presence of anti-phospholipid antibodies in APS pregnancies, and negatively correlates to pregnancy outcome. ${ }^{15}$ Moreover, a significant increase in antibody titles to a number of viruses, such as rubeovirus, measles, Parainfluenzavirus types 1, 2 and 3, Reovirus type 2, Epstein-Barr virus, mumps, HIV 1, and HCV has been reported in autoimmune diseases without any clinical evidence of infection. ${ }^{14}$ Furthermore, higher IgG concentrations against Toxoplasma gondii were found in patients with autoimmune thyroid diseases than in controls, suggesting that the protozoa may be involved in the initiation of both Hashimoto's thyroiditis and Grave's disease. ${ }^{6}$ When serological testing for CMV is considered, false-positive results for CMV IgM were described in autoimmune populations outside of pregnancy. ${ }^{9,16}$ A number of different mechanisms were suggested to explain these results, and many of them are not completely understood. A proposed mechanism is a non-specific and polyclonal stimulation of B lymphocytes with activation of memory cells reproducing anti-CMV IgM. This immune activation is more likely to occur in patients with autoimmune disease because of the dysregulation of their immune system. ${ }^{10}$ Another possible explanation is the presence of auto antibodies related to the autoimmune disease which can interfere with serological detection. ${ }^{10}$ Apart from the relationship between the autoimmune disease and false-positive tests for infections, the appearance of specific anti-CMV IgM can also be due to a heterotypical immune response caused by concurrent infections. In fact, polyclonal stimulation during acute Epstein-Barr virus infection is a well-known phenomenon. ${ }^{17-19}$ Antigenic cross-reactivity among the herpes viruses may also account for false positive serological results.

Several studies have observed that in autoimmune diseases the presence of anti-CMV IgM is associated with a worse evolution of the autoimmune disease itself. ${ }^{17} \mathrm{~A}$ few studies have focused on the possible relationship between false-positive results of the test for syphilis and pregnancy outcome in healthy women. ${ }^{20,21}$ In this report, there was a 14-fold difference in the incidence of false-positive anti-CMV IgM between pregnancies complicated by autoimmune disease and the control group, and false-positive anti-CMV was negatively related to pregnancy outcome. Autoimmune patients with false-positive anti-CMV IgM experienced a poor pregnancy outcome in comparison with patients without false-positive results. On the contrary, in low-risk pregnancies (control group), false positivity for anti-CMV IgM did not have the same significance observed in high-risk pregnancies (those complicated by autoimmune disease (study group)). In the study group, the worst pregnancy outcome was encountered in SLE patients with positive anti-CMV IgM. In the other subgroups, a detrimental effect of false positivity was observed, although it was less evident. The NPV for IUGR and neonatal birth weight $2000 \mathrm{~g}$ favors the test's reliability in reassuring patients, such as in situations warranting care management, as values of positive anti-CMV IgM screen test are in themselves poor at predicting negative pregnancy outcomes.

In our opinion, false-positive anti-CMV IgM in pregnant patients with autoimmune disease can be considered as the tip of the iceberg. In fact it may reflect a non-specific activation of the immune system that negatively affects pregnancy outcome. This 'immune damage' is independent of the presence of anti-phospholipid antibodies, as sug- gested by our findings. Therefore, when false-positive anti-CMV IgM is diagnosed, a strategy can be employed in the prenatal counseling of autoimmune pregnant patients. On the one hand, we can reassure the patient about the absent risk of congenital CMV infection. On the other hand, we can alert the patient about the increased obstetrical risk, because false positivity is related to poor pregnancy outcome. In conclusion, our results suggest that false-positive anti-CMV IgM in autoimmune patients could be considered an early prognostic factor of poor pregnancy outcome, in addition to well-known risk factors such as the presence of APS, hypertension, renal failure and disease activity. False positivity could be effective and helpful in identifying pregnancies at higher risk of obstetric complications, in order to recommend adequate prenatal care. Further prospective studies in a larger population with autoimmune disease should be performed to confirm our intriguing findings.

\section{Acknowledgements}

None.

\section{Conflicts on interest}

The authors declare that there have no conflicts of interest.

\section{Funding}

None.

\section{References}

1. Shoenfeld Y, Gilburd B, Abu-Shakara M, et al. The mosaic of autoimmunity: genetic factors involved in autoimmune diseases. Isr Med Assoc J. 2008;10(1):3-7.

2. Shoenfeld Y, Zandman-Goddard G, Stojanovich L, et al. The mosaic of autoimmunity: hormonal and environmental factors involved in autoimmune diseases. Isr Med Assoc J. 2008;10(1):8-12.

3. Cho JH, Gregersen PK. Genomics and the multifactorial nature of human autoimmune disease. $N$ Engl J Med. 2011;365(17):1612-1623.

4. Caldas CAM, de Carvalho JF. The role of environmental factors in the pathogenesis of non-organ-specific autoimmune diseases. Best Pract Res Clin Rheumatol. 2012;26(1):5-11.

5. Getts DR, Chastain EM, Terry RL, et al. Virus infection, antivira immunity, and autoimmunity. Immunol Rev. 2003;255(1):197-209.

6. Tozzoli R, Barzilai O, Ram M, et al. Infections and autoimmune thyroid diseases: parallel detection of antibodies against pathogens with proteomic technology. Autoimmun Rev. 2008;8(2):112-115.

7. Borg EJT, Hoarst G, Hummel E, et al. Rises in anti-double stranded DNA antibody levels prior to exacerbations of systemic lupus erythematosus are not merely due to polyclonal B cell activation. Clin Immunol Immunopathol. 1991;59(1):117-128.

8. Mendelson E, Aboudy Y, Smetana Z, et al. Laboratory assessment and diagnosis of congenital viral infections: Rubella, cytomegalovirus (CMV), varicella-zoster virus (VZV), herpes simplex virus (HSV), parvovirus B19 and human immunodeficiency virus (HIV). Reprod Toxicol. 2006;21(4):350-382.

9. Miendje Deyi Y, Goubau P, Bodéus M. False-positive IgM antibody tests for Cytomegalovirus in patients with acute Epstein-Barr virus infection. Eur J Clin Microbiol Infect Dis. 2000;19(7):557-560.

10. Cannavan FP, Costallat LT, Bertolo MB, et al. False positive IgM antibody tests for human cytomegalovirus (HCMV) in patients with SLE. Lupus. 1998;7(1):61-62. 
11. Munro SC, Hall B, Whybin LR, et al. Diagnosis of and screening for cytomegalovirus infection in pregnant women. J Clin Microbiol. 2005;43(9):4713-4718.

12. Haserick JR, Long R. Systemic lupus erythematosus preceded by falsepositive serologic tests for syphilis: presentation of five cases. Ann Intern Med. 1952;37(3):559-565.

13. De Carolis S, Santucci S, Botta A, et al. The relationship between TORCH complex false positivity and obstetric outcome in patients with antiphospholipid syndrome. Lupus. 2012;21(7):773-775.

14. Rothfield NF, Evans AS, Niederman JC. Clinical and laboratory aspects of raised virus antibody titres in systemic lupus erythematosus. Ann Rheum Dis. 1993;32(3):238-246.

15. Caruso A, De Carolis S, Ferrazzani S, et al. Pregnancy outcome in relation to uterine artery wave forms and clinical characteristics in women with antiphospholipid syndrome. Obstet Gynecol. 1993;82(6):970-977.

16. Stratta P, Colla L, Santi S, et al. IgM antibodies against cytomegalovirus in SLE nephritis: viral infection or a specific auto antibody? J Nephrol. 2002;15(1):88-92.
17. Moler E, Strom H, Al-Balaghi S. Role of polyclonal activation in specific immune responses. Relevance for findings of antibody activity in various diseases. Sc and J Immunol. 1980;12(3):177-182.

18. Rasmussen L, Kelsall D, Nelson R, et al. Virus-specific IgG and IgM antibodies in normal and immunocompromised subjects infected with cytomegalovirus. J Infect Dis. 1982;145(2):191-199.

19. Su BY, Su CY, Yu SF, et al. Incidental discovery of high systemic lupus erythematosus disease activity associated with cytomegalovirus viral activity. Med Microbiol Immunol. 2007;196(3):165-170.

20. Thornton JG, Foote GA, Page CE, et al False positive results of tests for syphilis and outcome of pregnancy. Br Med J. 1987;295(6594):355-356.

21. Henriksen R, Sogaard PE, Grennert L, et al. Autoimmune antibodies and pregnancy outcome in women with false-positive syphilis test results. A retrospective controlled investigation of women from 5170 deliveries. Acta Obstet Gynecol Scand. 1989;68(6):537-540. 